\title{
High Stability Performance of Superhydrophobic Modified Fluorinated Graphene Films on Copper Alloy Substrates
}

\author{
Rafik Abbas, N. Elkhoshkhany, Ahmed Hefnawy, Shaker Ebrahim, and Aya Rahal \\ Department of Materials Science, Institute of Graduate Studies and Research, Alexandria University, \\ 163 Horreya Avenue, Shatby 21526, Egypt \\ Correspondence should be addressed to Rafik Abbas; rafik.abbas@yahoo.com
}

Received 16 November 2016; Accepted 30 January 2017; Published 19 February 2017

Academic Editor: Mikhael Bechelany

Copyright (C) 2017 Rafik Abbas et al. This is an open access article distributed under the Creative Commons Attribution License, which permits unrestricted use, distribution, and reproduction in any medium, provided the original work is properly cited.

A stable self-cleaning superhydrophobic modified fluorinated graphene surface with micro/nanostructure was successfully fabricated on copper substrates via drop coating process. Irregularly stacked island-like multilayered fluorinated graphene nanoflakes comprised the microstructure. The fabricated films exhibited outstanding superhydrophobic property with a water contact angle $167^{\circ}$ and water sliding angle lower than $4^{\circ}$. The developed superhydrophobic surface showed excellent corrosion resistance with insignificant decrease of water contact angle $166^{\circ}$ in $3.5 \mathrm{wt} . \% \mathrm{NaCl}$ solution. This stable highly hydrophobic performance of the fluorinated graphene films could be useful in self-cleaning, antifogging, corrosion resistive coatings and microfluidic devices.

\section{Introduction}

Recent years have witnessed the increasing research interests in graphene and the graphene-related materials; graphene has revealed a cornucopia of both fundamental science and potential applications due to its ultra-high carrier mobility, optical transparency in the visible regime, high mechanical strength, high thermal conductivity, remarkable flexibility, exceptional thermal and chemical stability, and biocompatibility and being impermeable to molecules even as small as helium makes them most favorable for formation of passive layer to protect metals from oxidation and corrosion especially in severe marine environment [1-4].

Great efforts have also been devoted to the surface wettability control of graphene and its related materials, as a comprehensive understanding of the surface property of graphene is highly desired in both fundamental science and practical applications [1]. Self-cleaning superhydrophobic surfaces have a water contact angle greater than $150^{\circ}$ and a sliding angle less than $10^{\circ}$ [5]. On the basis of reducing surface energy and enhancing surface roughness [6], numerous artificial approaches of superhydrophobic surfaces have been developed by means of layer-by-layer assemblies [79], spraying [10], electrospinning [11, 12], spin-coating [13], electrochemical reaction and deposition [14-17], solution immersion process [18], drop coating [19], chemical vapor deposition [20], and so forth.

Graphene sheets (GS) are chemically inert, preventing any kind of interaction [21]. With a graphene plane decorated with hydroxyl, carbonyl, epoxy, and phenol functionalities, graphene oxide (GO) has shown remarkable promise as a manipulable precursor [22, 23]. GO is naturally hydrophilic, so in order to drastically change its surface wettability postfunctionalization of GO with hydrophobic groups seems a simple but effective method [1]. The prospect of having a graphitic basal plane with both oxygen and fluorine covalently bonded has a distinct set of advantages. The outstanding electronegativity of fluorine and the high polarity of the $\mathrm{C}-\mathrm{F}$ bond make it a superior alternative to the $\mathrm{C}-\mathrm{O}$ bond in GO. More importantly, the low surface energy of the C$\mathrm{F}$ bond allows for tailoring the wetting characteristics of $\mathrm{GO}$ and modifying its physiochemical properties [24, 25]. Wang et al. [26] developed an easy, low-cost, and effective synthesis route of fluorinated graphene (FG) through the reaction 
between dispersed GO and hydrofluoric acid (HF). The results suggested that the fluorination degree can be easily controlled. The as-prepared FG was difficult to be dispersed in water even after long-time ultrasonic treatment [26]. In this study, we report a feasible method to fabricate stable self-cleaning superhydrophobic and modified functionalized graphene coatings with nanoscaled surface roughness via a facile one-step process as a potential application to protect copper based structure from oxidation. The graphene coatings were fabricated via three steps: fabrication of GO, functionalizing graphene oxide with hydrofluoric acid, and modification of functionalized graphene films to lower the surface energy. The FG coating exhibits a superior surface roughness, which is crucial for its superhydrophobicity.

\section{Experimental}

2.1. Materials. Graphite powder (95.0\%) was purchased from Qualikems Fine Chemicals, India. Potassium permanganate $\left(\mathrm{KMnO}_{4}, 99.5 \%\right)$ was purchased from Alpha Chemika, India. Sodium nitrate $\left(\mathrm{NaNO}_{3}, 95.0 \%\right)$ was purchased from Chemajet, Egypt. Hydrofluoric acid (HF, 40.0\%) was purchased from Loba Chemie, India. $1 \mathrm{H}, 1 \mathrm{H}, 2 \mathrm{H}, 2 \mathrm{H}-$ Perfluorooctyltriethoxysilane (POTS, $\mathrm{C}_{14} \mathrm{H}_{15} \mathrm{~F}_{17} \mathrm{O}_{3} \mathrm{Si}$, 98\%) was purchased from Shanghai Xinda Chemical Industry, China. All chemical reagents used in the experiment were of analytical grade and used without further purification.

2.2. Preparation of Graphene Oxide. GO was synthesized using a modified Hummers method through graphite powder oxidation with sulfuric acid and potassium permanganate $\left(\mathrm{H}_{2} \mathrm{SO}_{4}-\mathrm{KMnO}_{4}\right)$. A detailed synthesis protocol has been reported [27].

2.3. Fluorination of Graphene Oxide. FG was synthesized by a simple hydrothermal reaction between GO dispersion and HF. FG was prepared by using three different times as discussed elsewhere [26]. Briefly, a sonicated HF-GO dispersion was transferred into a Teflon-lined autoclave and temperature was maintained at $180^{\circ} \mathrm{C}$ for 10,20 , and $30 \mathrm{~h}$. Thusly, FG 10, FG 20, and FG 30 were obtained.

2.4. Developing of Superhydrophobic Substrates. Copper alloy specimens were abraded using silicon carbide papers and then washed with ethanol and left to dry. Modification procedure was performed in either one step or two steps.

As for modifications in two-step process, substrates were fabricated with different concentrations of FG suspension in THF via drop coating process. POTS was applied via vapor deposition (VD) process. Substrates coated with FG were introduced into a chamber, and the POTS compound was placed on a hot plate so that it evaporates for $1 \mathrm{~h}$. Thusly, VFG 10, VFG 20, and VFG 30 were obtained.

As for modifications in one-step process, different concentrations of FG were added to solutions of $95 \mathrm{wt} . \%$ ethanol and $5 \mathrm{wt} . \%$ deionized water and $3 \mathrm{wt} . \%$ POTS. The suspensions were stirred for $2 \mathrm{~h}$ and then left overnight. The solutions of modified FG powder and THF were sonicated

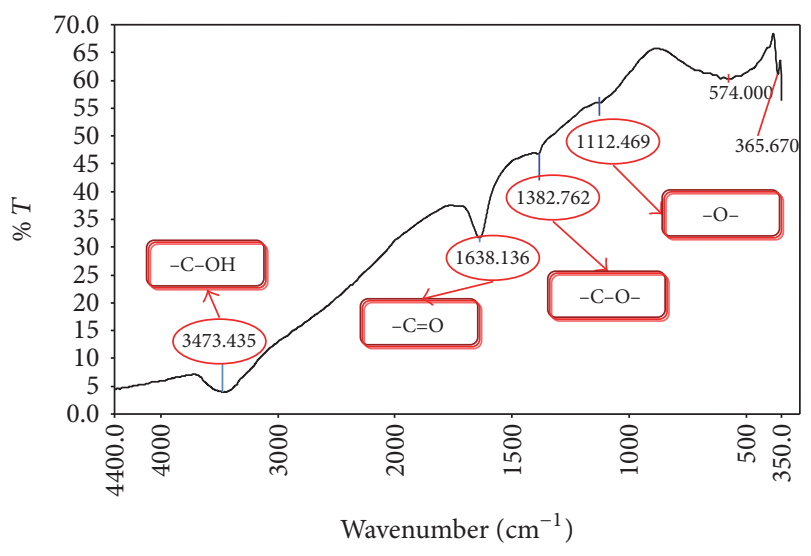

FIGURE 1: FTIR spectra of GO powder.

for $5 \mathrm{~min}$ and then applied via drop coating. Thusly, DFG 10, DFG 20, and DFG 30 were obtained.

2.5. Sample Characterization. The surface morphology of the superhydrophobic copper alloy substrates was characterized by scanning electron microscopy (SEM, JSM-6390A). X-ray diffraction analysis of GO powder was carried out by X-ray diffraction microscopy (XRD, Schimad Zn 7000). The optical absorption of the FG powder was measured by a UV-visible spectrophotometer (UV, Analytic Jena AG, Specord 50). The infrared transmission spectra were recorded at room temperature on Fourier-transform infrared spectrophotometer (FTIR, Bruker-Tensor 27). The spectra were collected over the range of $500-4,400 \mathrm{~cm}^{-1}$. The surface wettability was assessed by contact angle (CA) measurements using Gaosuo USB digital microscope (magnification range $1 \mathrm{x} \sim 500 \mathrm{x}$ ); all the angles were determined by averaging values measured at five points on different locations on each sample surface. The corrosion resistance of the samples was examined via potentiodynamic polarization curves and electrochemical impedance spectroscopy (EIS) acquired by electrochemical experiments in $3.5 \mathrm{wt} . \% \mathrm{NaCl}$ solution. Electrochemical experiments were performed using a computer-controlled electrochemical system (GAMRY PCI4G750 instruments) at room temperature. Moreover, the adhesion of the superhydrophobic coatings on copper substrate was carried out according to the ISO-2409 standard Tape Test method using Cross Hatch Cutter, model Elcometer 107.

\section{Results and Discussion}

3.1. Characterization of GO and FG. In this study, FTIR and XRD analyses were used to confirm formation of GO. FTIR is a standard technique used to identify the type of oxygen functionalities and bonding configuration in GO. Figure 1 displays the IR spectrum of GO, prepared via the modified Hummers method. The FTIR spectra of GO possess four main peaks [28]. The predominant characteristic absorption signals show in Figure 1, firstly, a broad and intense peak at approximately $3473 \mathrm{~cm}^{-1}$ attributed to $(\mathrm{O}-\mathrm{H})$ stretching of carboxyl groups. Secondly, the one centered at approximately 


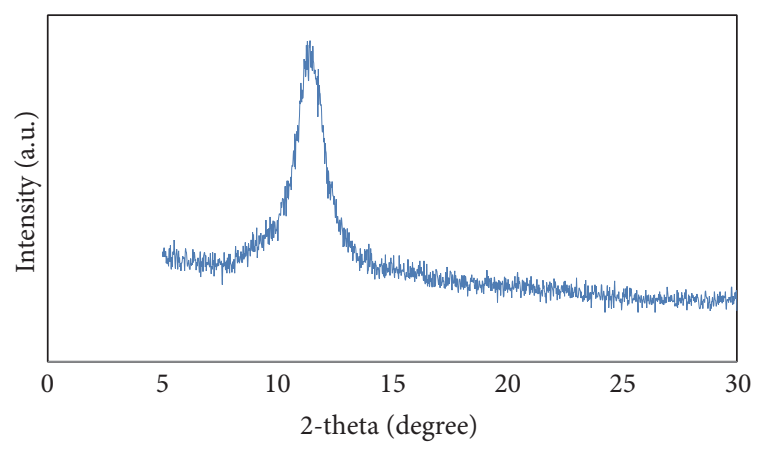

FIGURE 2: XRD spectra of GO powder.
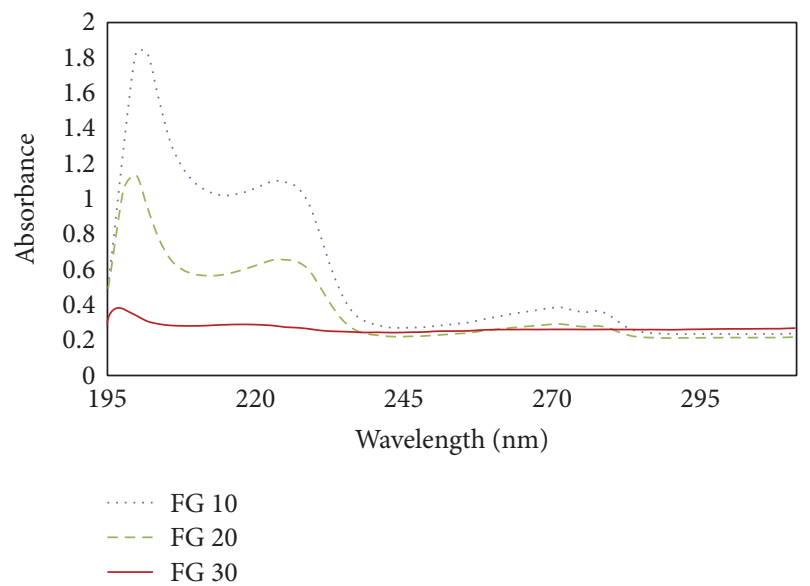

Figure 3: UV-vis absorption spectra of FG 10, FG 20, and FG 30.

$1638 \mathrm{~cm}^{-1}$ corresponds to the vibrational mode of the ketone groups $(-\mathrm{C}=\mathrm{O})$. Another peak at approximately $1382 \mathrm{~cm}^{-1}$ is assigned to a $(\mathrm{C}-\mathrm{O})$ vibrational mode. Finally, the peak at approximately $1112 \mathrm{~cm}^{-1}$ arises from epoxide groups (C-OC). Peaks below $900 \mathrm{~cm}^{-1}$ are not usually interpreted because they represent too complex a structural signature [28].

$\mathrm{XRD}$ is another useful technique to reveal the interlayer distances, crystalline structure, and layer numbers in the solid form of a material [29]. The XRD spectra support the order of overall oxidation. For XRD, the interlayer spacing of the materials is proportional to the degree of oxidation [30]. Figure 2 shows the XRD spectra in which the GO diffraction peak occurs at $11.4^{\circ}$, indicating the presence of oxygen containing functional groups after the oxidation process while the spacing of GO was $7.7 \AA$.

$\mathrm{UV}$-vis spectroscopy has been proven to be an effective optical characterization tool for demonstrating the different FG degrees of fluorination. The optical absorption spectra of FG 10, FG 20, and FG 30 were recorded, and the results were shown in Figure 3. The three spectra were recorded for identical concentration of each material. The degree of remaining conjugation can be determined by $\lambda_{\max }$ of each $\mathrm{UV}$-vis spectrum. The more $\pi \rightarrow \pi^{*}$ transitions (conjugation), the less energy needs to be used for the electronic transition, which results in a higher $\lambda_{\max }$ [30]. Wang et al. [26] presented that the optical absorbance feature of FG is changed

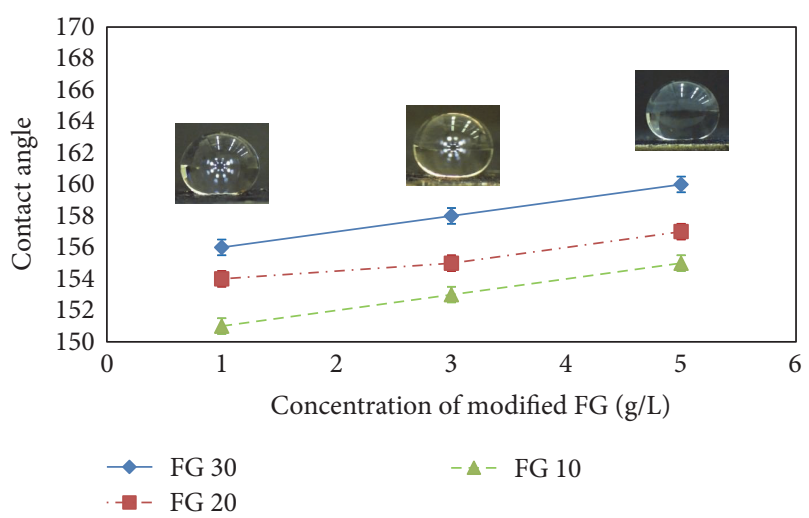

FIGURE 4: The relationship between the contact angle of the modified FG substrates and the different concentration of modified FG suspension for 1,3 , and $5 \mathrm{~g} / \mathrm{l}$ at different fluorination degrees VFG 10, VFG 20, and VFG 30.

remarkably by fluorination, which causes the appearance of an additional peak at 200 and $198 \mathrm{~nm}$.

3.2. Wettability Studies. To determine the superhydrophobicity of these films, water contact angle (WCA) measurements were performed at ambient conditions. The surface wetting property of the superhydrophobic coat depends on chemical composition, surface free energy, and surface morphology; consequently they have an important role in attaining the desired wettability.

For an absolutely flat surface, wettability is determined by the surface free energy of the solid described by Young's equation but it is not the only factor. For a real surface that is usually rough, topological roughness plays a critical role in the wetting behavior of solid surfaces described by two classical empirical models, Wenzel regime and Cassie-Baxter regime [1].

Mathkar et al. explained using Young's equation that adding the high polar and the low surface energy $\mathrm{C}-\mathrm{F}$ bonds leads to the increase of the superhydrophobic property of FG, as minimizing the surface free energy leads to the highest possible contact angle, depending on the surface tension of the liquid [22].

Bharathidasan et al. explained the transition from the Wenzel regime to Cassie regime for the nonwetting and selfcleaning surfaces with WCA of $150.2^{\circ}$ and sliding angle (SA) $<12^{\circ}$, as the roughness created on the surface of FG might be sufficient to trap air inside the voids of the surface causing a heterogeneous surface of both air and solid, which reduces the adhesive force between the water and solid surface and for this case contact angle is to be described in terms of CassieBaxter equation [31].

The surface wettability of the superhydrophobic substrates was affected impressively by the degree of fluorination, surface modification, and the concentration of FG suspension. Figure 4 demonstrates the relationship between the contact angle of the superhydrophobic substrates and the different concentration of modified FG suspension for 1,3 , and $5 \mathrm{~g} / \mathrm{l}$ at different fluorination degrees VFG 10, VFG 


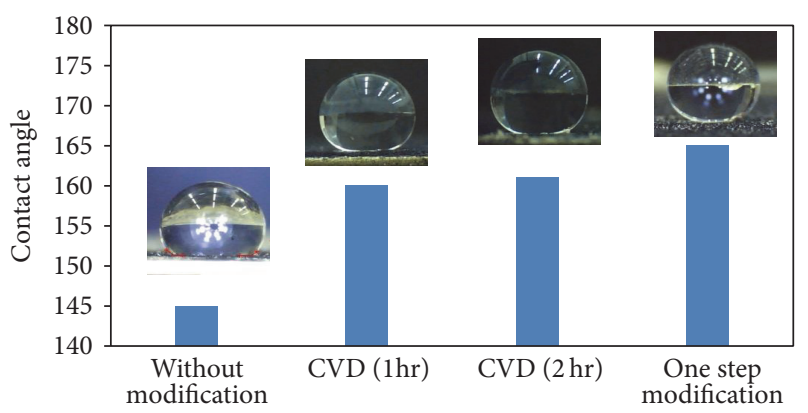

Figure 5: The effect of different modification techniques on contact angle for FG 30.

20, and VFG 30. As indicated in Figure 4, by increasing fluorination degrees of modified FG, WCA increases. Also, as the concentration of modified FG increases, WCA increases. VFG 30 exhibited the highest WCAs while $5 \mathrm{~g} / \mathrm{l}$ concentrations hold the highest WCAs of all the concentrations we used. This indicates that tailoring wetting characteristics of FG depends on fluorine which is the most electronegative element in existence (Young's regime).

Figure 5 illustrates the different modification techniques effect on the WCA performed on substrates of FG 30 and $5 \mathrm{~g} / \mathrm{l}$. WCA was greatly improved by modification; FG without modification acquired a WCA of $145^{\circ}$. Modification increases the presence of fluorine atoms in the structure causing the reduction in the surface energy of FG. As for the technique of modification, one-step modification of FG exhibited an enhancement owing to the direct interaction between POTS and FG. The difference of WCA exposed that one-step modification of FG not only eliminates complexity and time consumption and reduced the energy consumed in VD process but also improved the superhydrophobicity by nearly 5 degrees.

3.3. Surface Morphology. Surface morphology is an important parameter of superhydrophobic properties; therefore, coatings were characterized by scanning electron microscopes (SEM). Contrasting to GO films [19], the modified FG substrates exhibited a superior surface roughness and sustained their roughness after corrosion. The derivation of the superior surface roughness of FG can be explained in terms of dispersion behaviors. GO is well dispersed in polar solvents nothing like FG as its hydrophobic nature leads to poor dispersion in polar solvents and the development of aggregated dominions. The morphology evolution and superhydrophobicity of the as-prepared modified FG substrates are clearly shown by the SEM images and water contact angle for concentrations of $5 \mathrm{~g} / \mathrm{l}$ solution of FG. Figure 6(a) displayed the SEM image of the achieved surface of VFG 10. The surface showed that there were islands and cavities existing distributed uniformly across the surface and making the surface roughness. The prepared surfaces exhibited micro- and nanoscale hierarchical structures, which had great cavities and surface roughness. Air could be trapped easily within the structure, so the WCA value of the surfaces was high (Cassie-Baxter regime). These microstructures on the substrate surface were irregular micro/nanostructures. Through further increase in the degree of fluorination the micro/nanostructures intensified. As shown in Figure 6(b) the surface of VFG 30 presented more micro/nanostructures. As for one-step modification, DFG 30 showed the highest degree of micro/nanostructures shown in Figure 6(c) possessing a CA of $167^{\circ}$; even after corrosion simulating test in saline environment of $3.5 \% \mathrm{NaCl}$ solution it preserved its surface micro/nanostructures as shown in Figure 6(d) with a CA of $166^{\circ}$. From both surface wettability and surface morphology increasing the degree of fluorination as well as using direct one-step modification process exceedingly increased the superhydrophobicity of the surfaces achieved.

It is important to mention that the mechanical properties of the superhydrophobic coatings are very important for their uses against surface erosion and friction, as well as corrosion protection [32]. Durability plays an essential role in real application [33]. In this study, adhesion strength of different prepared superhydrophobic copper alloy surfaces was evaluated using the Tape Test (ISO-2409: Standard Test Methods for Measuring Adhesion by Tape Test). Our results showed that the superhydrophobic surfaces ensured a good adhesion property. The edges of the indented squares were utterly smooth and had not detached after applying the tap. According to the ISO standard, the coatings were classified as zero grade. All the droplets showed spherical shape. The value of WCA was not affected after adhesion test. This indicated that the superhydrophobicity was not affected by the test.

\subsection{Electrochemical Measurements and Corrosion Inhibition Performance}

3.4.1. Electrochemical Impedance Spectroscopy. The electrochemical impedance spectroscopy (EIS) was applied for understanding electrochemical degradation of uncoated and the coated substrates of copper alloy. EIS was applied to explore the corrosion resistance ability of the superhydrophobic coatings in saline environment of $3.5 \mathrm{wt} . \% \mathrm{NaCl}$ aqueous solution. Figure 7 showed the EIS results in the form of Nyquist plots in which the imaginary impedance $\left(Z_{\text {im }}\right)$ was plotted against the real impedance $\left(Z_{\mathrm{re}}\right)$. For comparison, the differences in the EIS curves between the uncoated substrate (Cu-bare) and the superhydrophobic substrates were tested.

The equivalent circuits, as shown in Figure 8, were used to consider all the process involved in the electrical response of the system. $R_{s}$ represents the solution resistance, $R_{p}$ is the polarization resistance and can be defined also as the chargetransfer resistance [3], and $W$ is the Warburg impedance. The different elements were evaluated by a fitting procedure.

Figure 7 shows that the Nyquist plots of coated samples are composed of a capacitive loop in high frequency range and a straight line (Warburg impedance) in low frequency range. The parameters obtained by fitting EIS data by using the equivalent circuits and the calculated protection efficiencies $(\% P)$ for uncoated and coated $\mathrm{Cu}$ alloy substrates at different conditions are listed in Table 1. It can be found that $R_{p}$ for coated copper samples is higher than that for uncoated copper sample and the protection efficiency is close to $97 \%$. 
TABLE 1: Electrochemical parameters obtained from Nyquist plots of uncoated and coated copper alloy with superhydrophobic FG.

\begin{tabular}{|c|c|c|c|c|c|c|}
\hline Substrate & $R_{P}\left(\Omega \cdot \mathrm{cm}^{2}\right)$ & $R_{S}\left(\Omega \cdot \mathrm{cm}^{2}\right)$ & $Y_{0}\left(S * s^{a}\right)$ & $\alpha$ & $\begin{array}{c}W_{d} \\
S * s^{(1 / 2)}\end{array}$ & $\% P$ \\
\hline Cu-bare & 239.7 & 19.71 & $11.84 * 10^{-3}$ & $492 * 10^{-3}$ & - & - \\
\hline VFG 10 & 3900 & 79.23 & $32.28 * 10^{-6}$ & $411 * 10^{-3}$ & $23.2 * 10^{-6}$ & 93.9 \\
\hline VFG 20 & 6600 & 95.59 & $27.51 * 10^{-6}$ & $385 * 10^{-3}$ & $15.3 * 10^{-6}$ & 96.4 \\
\hline VFG 30 & 4300 & 84.21 & $30.24 * 10^{-6}$ & $521 * 10^{-3}$ & $17.9 * 10^{-6}$ & 94.4 \\
\hline DFG 30 & 6500 & 97.38 & $20.2 * 10^{-6}$ & $399 * 10^{-3}$ & $9.5 * 10^{-6}$ & 96.3 \\
\hline
\end{tabular}
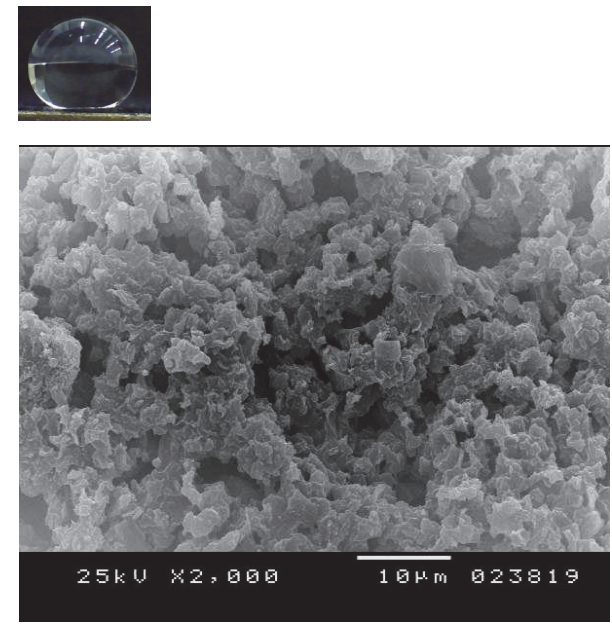

(a)
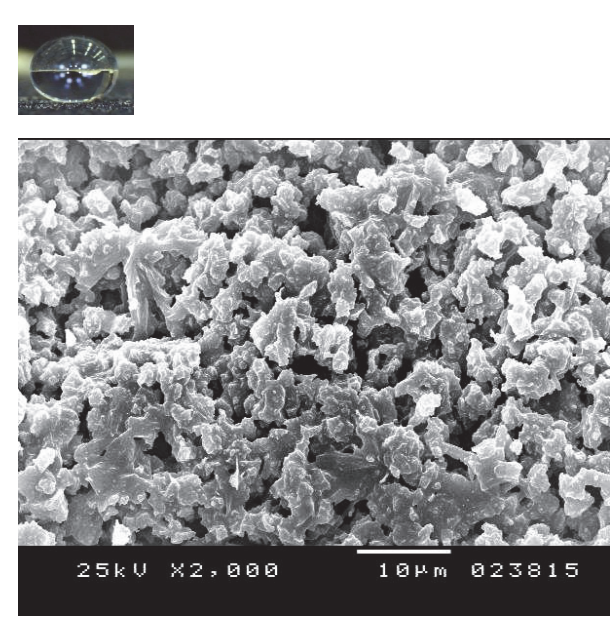

(c)

\section{$6^{1 \%}$}

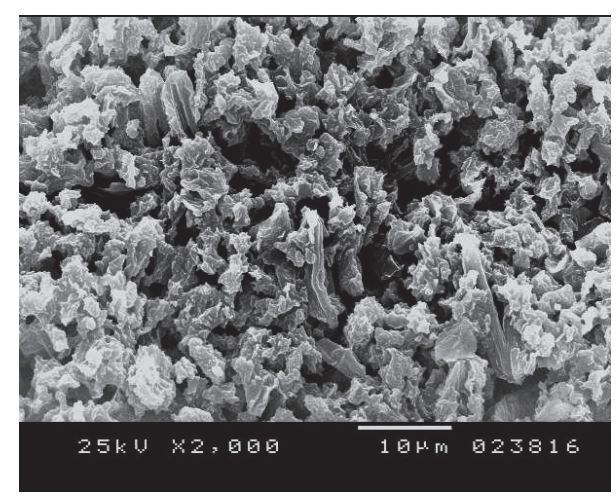

(b)
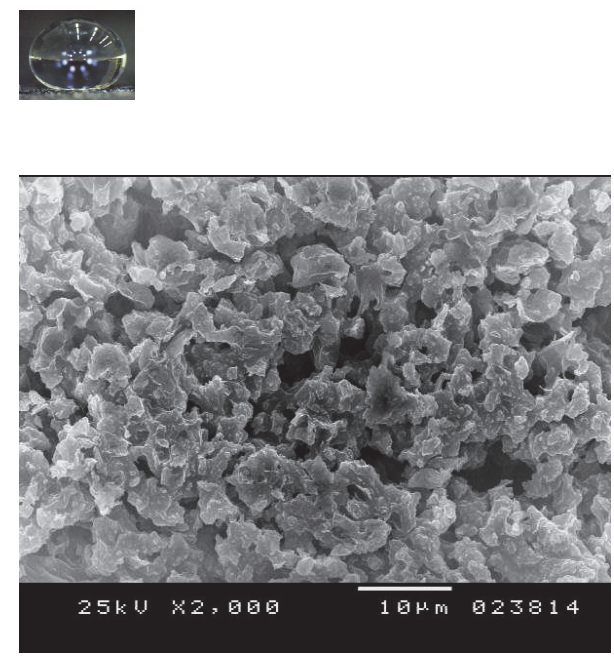

(d)

FIGURE 6: SEM images of modified FG on copper alloy surfaces (a) VFG 10, (b) VFG 30, (c) DFG 30 before corrosion, and (d) DFG 30 after corrosion.

3.4.2. Potentiodynamic Polarization. The polarization studies were carried out to assess the relative susceptibility of superhydrophobic modified FG coatings to corrosion, under aggressive electrolyte environment. Figure 9 shows typical polarization curves for copper with different superhydrophobic modified FG coatings in chloride media. Corrosion potential $\left(E_{\text {corr }}\right)$ and corrosion current density $\left(i_{\text {corr }}\right)$ are used to evaluate the corrosion resistance property of the coatings; they were extracted from the intercept of Tafel slopes using Tafel extrapolation method and were summarized in Table 2.

As shown in Figure 9 compared with the uncoated $\mathrm{Cu}$ substrate, all the coated substrates exhibited a drastic shift of corrosion potential $\left(E_{\text {corr }}\right)$, thus revealing the improved corrosion resistance of the coated substrates. Remarkably, the corrosion current densities $\left(i_{\text {corr }}\right)$ of superhydrophobic modified FG coatings were expressively lower than the bare 
TABLE 2: Electrochemical parameters obtained from potentiodynamic polarization of uncoated and coated copper alloy with superhydrophobic FG.

\begin{tabular}{|c|c|c|c|c|c|}
\hline Substrate & $I_{\text {corr. }}(\mu \mathrm{A})$ & $-E_{\text {corr. }}(\mathrm{mV})$ & $\beta_{a}(\mathrm{mV} /$ decade $)$ & $\beta_{c}(\mathrm{mV} /$ decade $)$ & $\% P$ \\
\hline Cu-bare & 93.20 & 268 & $59.6 * 10^{-3}$ & $216 * 10^{-3}$ & - \\
\hline VFG 10 & 0.29 & 475 & $051 * 10^{-3}$ & $152 * 10^{-3}$ & 99.60 \\
\hline VFG 20 & 0.16 & 494 & $54.9 * 10^{-3}$ & $063 * 10^{-3}$ & 99.80 \\
\hline VFG 30 & 0.13 & 467 & $057 * 10^{-3}$ & $265 * 10^{-3}$ & 99.85 \\
\hline DFG 30 & 0.09 & 489 & $072 * 10^{-3}$ & $081 * 10^{-3}$ & 99.90 \\
\hline
\end{tabular}

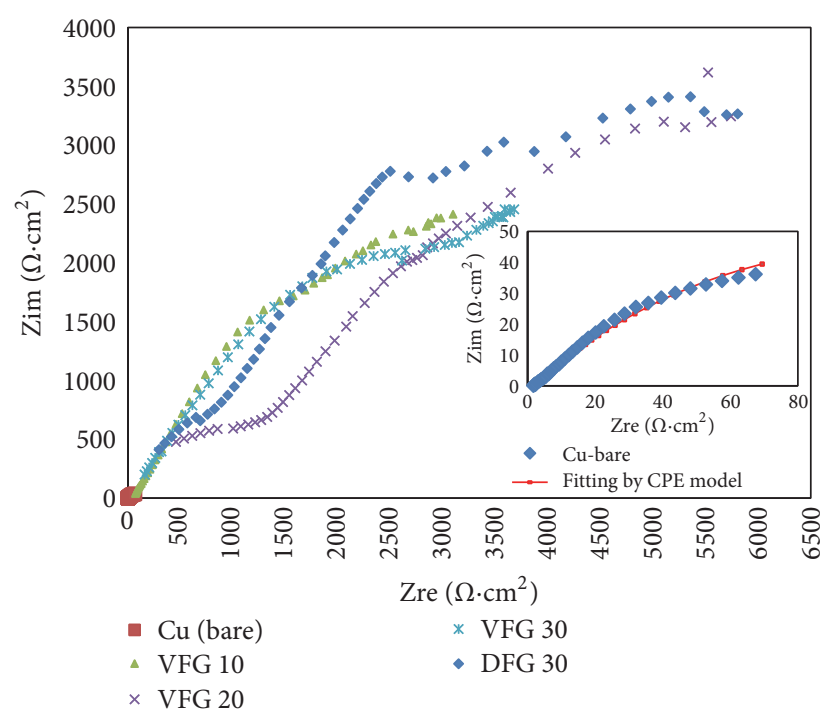

FIGURE 7: Nyquist plots for copper substrate and the superhydrophobic surfaces prepared with different degrees of fluorination and modification techniques in $3.5 \mathrm{wt} . \% \mathrm{NaCl}$ solution.

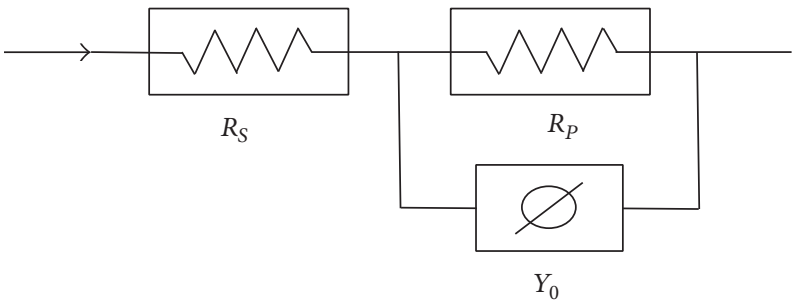

(a)

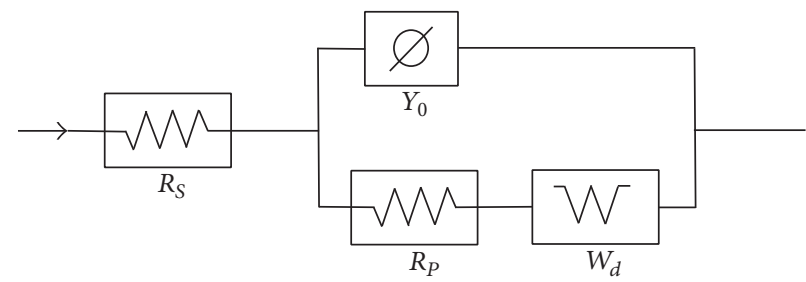

(b)

FIGURE 8: Equivalent circuits models used in the fitting of the impedance data of copper (a) without and (b) with coating in 3.5 wt.\% NaCl solution.

$\mathrm{Cu}$. This establishes the robust corrosion resistance of superhydrophobic modified FG coatings in saline environment. Generally, the modified copper surfaces with superhydrophobic modified FG layer demonstrate higher protection against corrosion. In addition, using direct modification technique was seen to be more effective than using vapor deposition modification technique as it acquired higher inhibition efficiency and superhydrophobicity.

\section{Conclusion}

We demonstrated simple and stable self-cleaning superhydrophobic modified fluorinated graphene surfaces fabricated on copper substrates via drop coating process. One-step modification of FG 30 exhibited the highest CA of $167^{\circ}$; even after corrosion simulating test in saline environment of $3.5 \%$ $\mathrm{NaCl}$ solution it preserved its surface micro/nanostructures with a CA of $166^{\circ}$. Consequently, this technique should be easily applied to large-scale manufacture of superhydrophobic engineering materials.

\section{Additional Points}

Research Highlights. (i) Superhydrophobic graphene is fabricated by simple and environment-friendly method. (ii) The coating remained stable after adhesion and corrosion tests. 


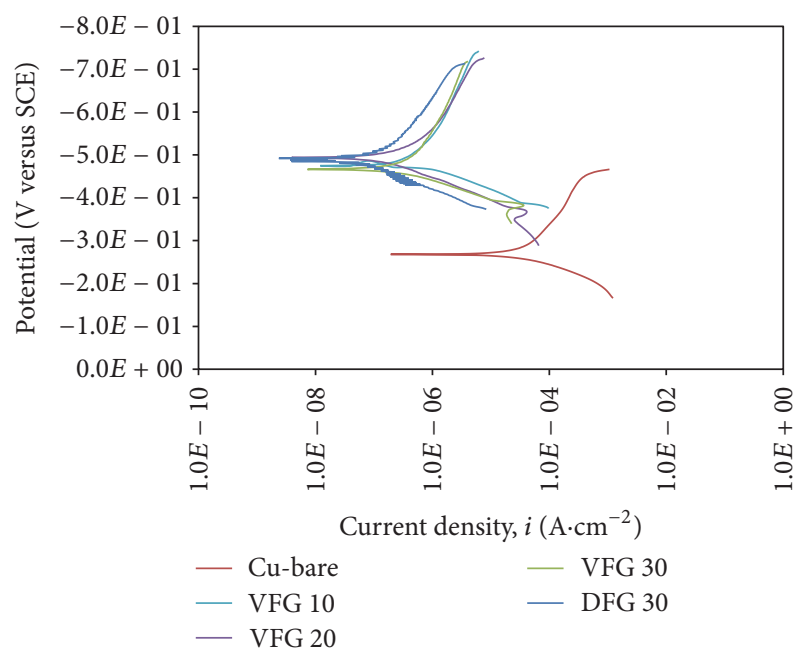

FIGURE 9: Potentiodynamic polarization curves of copper substrate and the superhydrophobic surfaces prepared with different degrees of fluorination and modification techniques in $3.5 \mathrm{wt} . \% \mathrm{NaCl}$ solution.

(iii) The corrosion rate decreases with the increase of the water contact angle. (iv) The superhydrophobic copper alloy has better corrosion resistance.

\section{Competing Interests}

The authors declare that they have no competing interests.

\section{References}

[1] J.-N. Wang, Y.-L. Zhang, Y. Liu, W. Zheng, L. P. Lee, and H.B. Sun, "Recent developments in superhydrophobic graphene and graphene-related materials: from preparation to potential applications," Nanoscale, vol. 7, no. 16, pp. 7101-7114, 2015.

[2] B. P. Singh, S. Nayak, K. K. Nanda, B. K. Jena, S. Bhattacharjee, and L. Besra, "The production of a corrosion resistant graphene reinforced composite coating on copper by electrophoretic deposition," Carbon, vol. 61, pp. 47-56, 2013.

[3] Y. Liu, J. Zhang, S. Li, Y. Wang, Z. Han, and L. Ren, "Fabrication of a superhydrophobic graphene surface with excellent mechanical abrasion and corrosion resistance on an aluminum alloy substrate," RSC Advances, vol. 4, no. 85, pp. 45389-45396, 2014.

[4] J. S. Bunch, S. S. Verbridge, J. S. Alden et al., "Impermeable atomic membranes from graphene sheets," Nano Letters, vol. 8, no. 8, pp. 2458-2462, 2008.

[5] N. Mittal, D. Deva, R. Kumar, and A. Sharma, "Exceptionally robust and conductive superhydrophobic free-standing films of mesoporous carbon nanocapsule/polymer composite for multifunctional applications," Carbon, vol. 93, pp. 492-501, 2015.

[6] T. Ning, W. Xu, and S. Lu, "Fabrication of superhydrophobic surfaces on zinc substrates and their application as effective corrosion barriers," Applied Surface Science, vol. 258, no. 4, pp. 1359-1365, 2011.
[7] X. Li, X. Du, and J. He, "Self-cleaning antireflective coatings assembled from peculiar mesoporous silica nanoparticles," Langmuir, vol. 26, no. 16, pp. 13528-13534, 2010.

[8] Y. Li, F. Liu, and J. Sun, "A facile layer-by-layer deposition process for the fabrication of highly transparent superhydrophobic coatings," Chemical Communications, no. 19, pp. 2730-2732, 2009.

[9] J. Bravo, L. Zhai, Z. Wu, R. E. Cohen, and M. F. Rubner, “Transparent superhydrophobic films based on silica nanoparticles," Langmuir, vol. 23, no. 13, pp. 7293-7298, 2007.

[10] Y. C. Jung and B. Bhushan, "Mechanically durable carbon nanotube composite hierarchical structures with superhydrophobicity self-cleaning and low-drag," ACS Nano, vol. 3, pp. 41554163, 2009.

[11] F. Li, Q. Li, and H. Kim, "Spray deposition of electrospun $\mathrm{TiO}_{2}$ nanoparticles with self-cleaning and transparent properties onto glass," Applied Surface Science, vol. 276, pp. 390-396, 2013.

[12] U. Cengiz, M. Z. Avci, H. Y. Erbil, and A. S. Sarac, "Superhydrophobic terpolymer nanofibers containing perfluoroethyl alkyl methacrylate by electrospinning," Applied Surface Science, vol. 258, no. 15, pp. 5815-5821, 2012.

[13] C. Kosak Söz, E. Yilgör, and I. Yilgör, "Influence of the average surface roughness on the formation of superhydrophobic polymer surfaces through spin-coating with hydrophobic fumed silica," Polymer, vol. 62, pp. 118-128, 2015.

[14] Q. Liu, D. Chen, and Z. Kang, "One-Step electrodeposition process to fabricate corrosion-resistant superhydrophobic surface on magnesium alloy," ACS Applied Materials and Interfaces, vol. 7, no. 3, pp. 1859-1867, 2015.

[15] P. Wang, D. Zhang, and Z. Lu, "Advantage of super-hydrophobic surface as a barrier against atmospheric corrosion induced by salt deliquescence," Corrosion Science, vol. 90, pp. 23-32, 2015.

[16] S. Khorsand, K. Raeissi, and F. Ashrafizadeh, "Corrosion resistance and long-term durability of super-hydrophobic nickel film prepared by electrodeposition process," Applied Surface Science, vol. 305, pp. 498-505, 2014.

[17] Z. Chen, L. Hao, and C. Chena, "Simultaneous fabrication of superhydrophobic coatings on cathodic and anodic copper surfaces with micro/nano-structures," ECS Electrochemistry Letters, vol. 1, no. 4, pp. D21-D23, 2012.

[18] F. Zhang, S. Chen, L. Dong, Y. Lei, T. Liu, and Y. Yin, "Preparation of superhydrophobic films on titanium as effective corrosion barriers," Applied Surface Science, vol. 257, no. 7, pp. 2587-2591, 2011.

[19] Y. Zhou, F. Xu, G. Jiang et al., "Superhydrophobic and high adhesive performance of functionalized graphene films," Powder Technology, vol. 230, pp. 247-251, 2012.

[20] S. Rezaei, I. Manoucheri, R. Moradian, and B. Pourabbas, "One-step chemical vapor deposition and modification of silica nanoparticles at the lowest possible temperature and superhydrophobic surface fabrication," Chemical Engineering Journal, vol. 252, pp. 11-16, 2014.

[21] B. P. Singh, B. K. Jena, S. Bhattacharjee, and L. Besra, "Development of oxidation and corrosion resistance hydrophobic graphene oxide-polymer composite coating on copper," Surface and Coatings Technology, vol. 232, pp. 475-481, 2013.

[22] A. Mathkar, T. N. Narayanan, L. B. Alemany et al., "Synthesis of fluorinated graphene oxide and its amphiphobic properties," Particle and Particle Systems Characterization, vol. 30, no. 3, pp. 266-272, 2013. 
[23] M. Li, Q. Liu, Z. Jia et al., "Electrophoretic deposition and electrochemical behavior of novel graphene oxide-hyaluronic acid-hydroxyapatite nanocomposite coatings," Applied Surface Science, vol. 284, pp. 804-810, 2013.

[24] C. Galande, W. Gao, A. Mathkar et al., "Science and engineering of graphene oxide," Particle and Particle Systems Characterization, vol. 31, no. 6, pp. 619-638, 2014.

[25] S. K. Yadav, J. H. Lee, H. Park, S. M. Hong, T. H. Han, and C. M. Koo, "Facile and ecofriendly fluorination of graphene oxide," Bulletin of the Korean Chemical Society, vol. 35, no. 7, pp. 21392142, 2014.

[26] Z. Wang, J. Wang, Z. Li et al., "Synthesis of fluorinated graphene with tunable degree of fluorination," Carbon, vol. 50, no. 15, pp. 5403-5410, 2012.

[27] T. Rattana, S. Chaiyakun, N. Witit-anun et al., "Preparation and characterization of graphene oxide nanosheets," Procedia Engineering, vol. 32, pp. 759-764, 2012.

[28] D. W. Lee, V. L. De Los Santos, J. W. Seo et al., "The structure of graphite oxide: investigation of its surface chemical groups," Journal of Physical Chemistry B, vol. 114, no. 17, pp. 5723-5728, 2010.

[29] S. Y. Toh, K. S. Loh, S. K. Kamarudin, and W. R. W. Daud, "Graphene production via electrochemical reduction of graphene oxide: synthesis and characterisation," Chemical Engineering Journal, vol. 251, pp. 422-434, 2014.

[30] D. C. Marcano, D. V. Kosynkin, J. M. Berlin et al., "Improved synthesis of graphene oxide," ACS Nano, vol. 4, no. 8, pp. 48064814, 2010.

[31] T. Bharathidasan, T. N. Narayanan, S. Sathyanaryanan, and S. S. Sreejakumari, "Above 170。 water contact angle and oleophobicity of fluorinated graphene oxide based transparent polymeric films," Carbon, vol. 84, no. 1, pp. 207-213, 2015.

[32] Y. Huang, D. K. Sarkar, D. Gallant, and X.-G. Chen, "Corrosion resistance properties of superhydrophobic copper surfaces fabricated by one-step electrochemical modification process," Applied Surface Science, vol. 282, pp. 689-694, 2013.

[33] Z. Zhang, B. Ge, X. Men, and Y. Li, "Mechanically durable, superhydrophobic coatings prepared by dual-layer method for anti-corrosion and self-cleaning," Colloids and Surfaces A: Physicochemical and Engineering Aspects, vol. 490, pp. 182-188, 2016. 

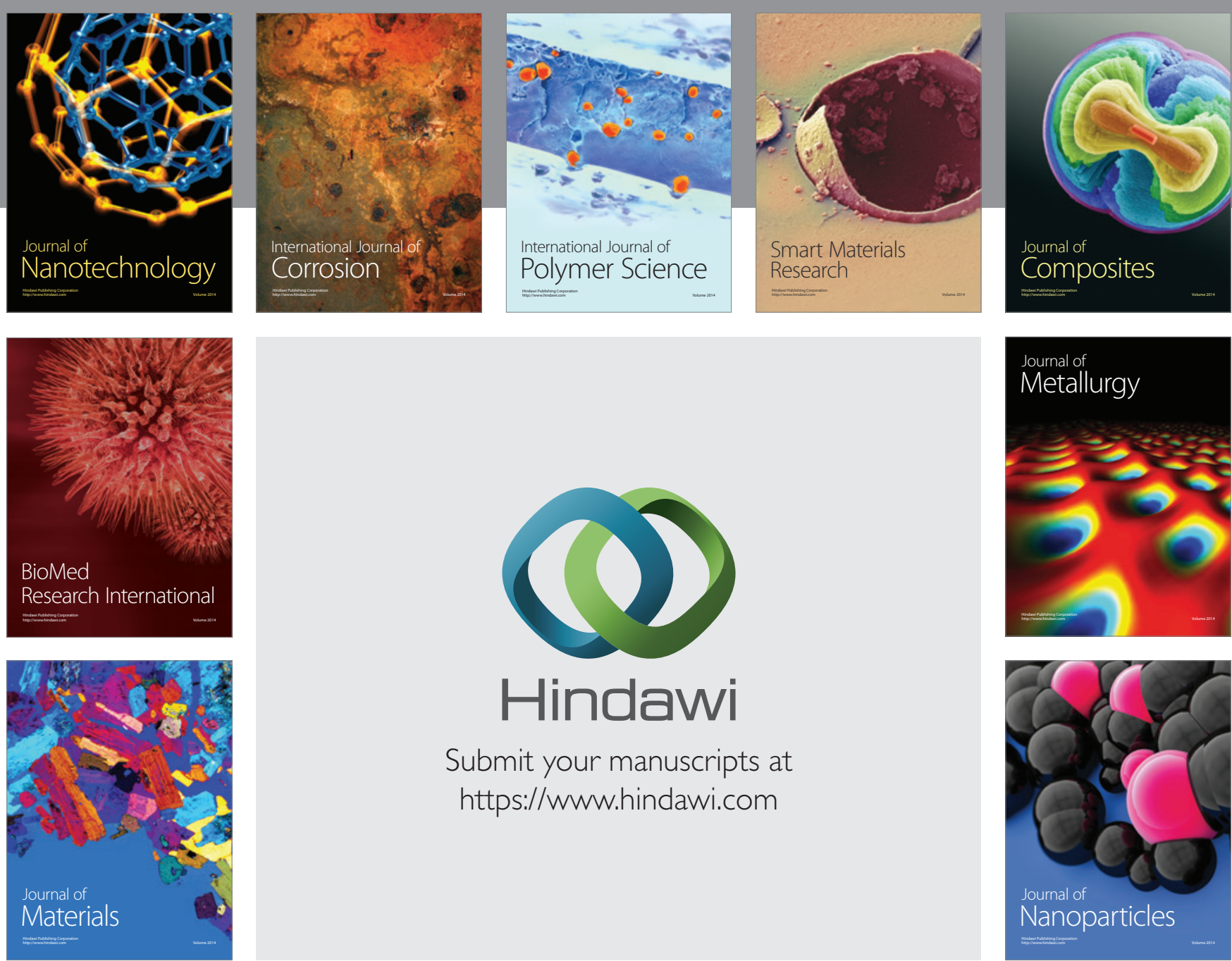

\section{Hindawi}

Submit your manuscripts at

https://www.hindawi.com

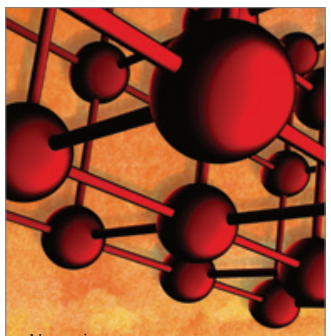

Materials Science and Engineering
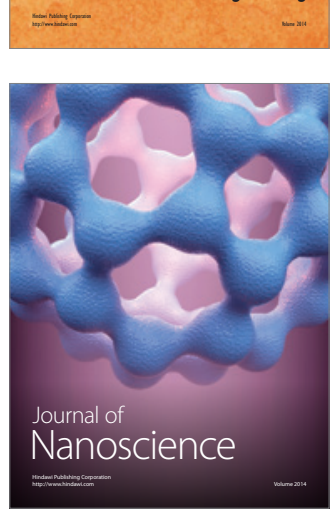
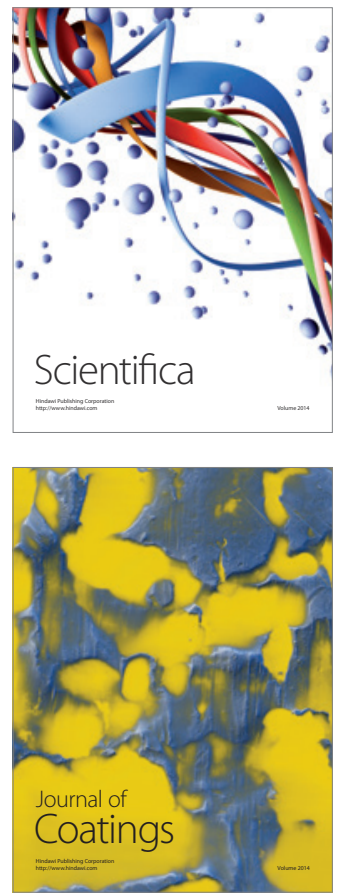
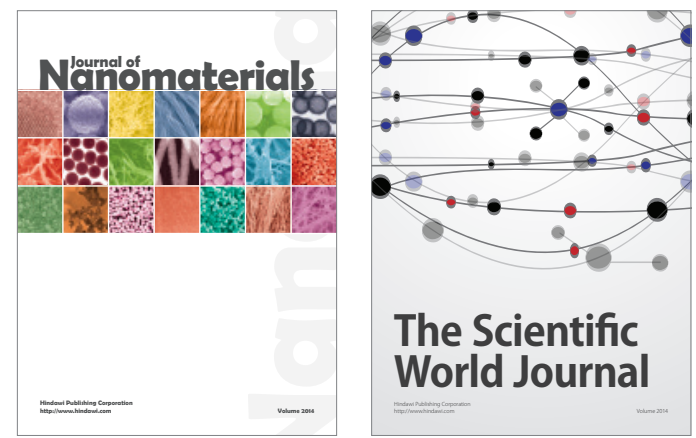

The Scientific World Journal
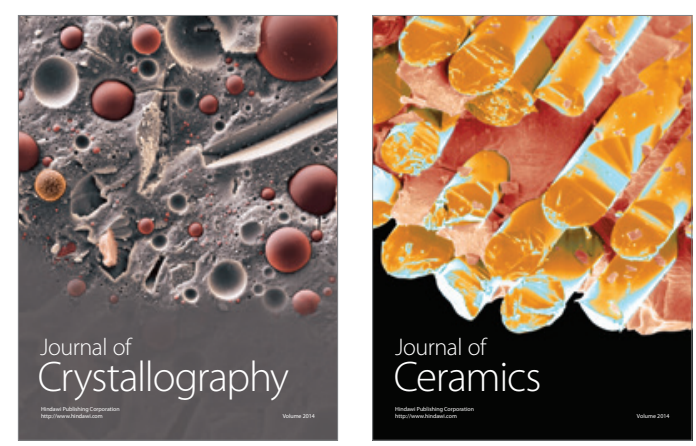
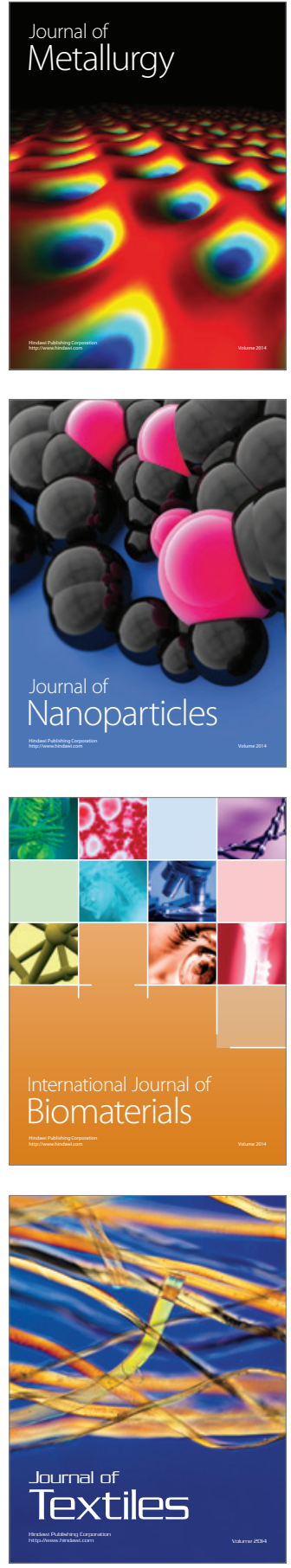